\title{
Effect of particle orientation on the diffusion of water in the dual porosity of vermiculite porous media
}

\author{
MR. ALI ASAAD, PHD $^{1}$, FABIEN HUBERT ${ }^{1}$, ERIC \\ FERRAGE $^{2}$, THOMAS DABAT ${ }^{3}$, ERWAN NICOLAS \\ PAINEAU $^{4}$, SEBASTIEN SAVOYE $^{5}$, BRIAN GREGOIRE ${ }^{3}$ \\ AND EMMANUEL TERTRE 6 \\ ${ }^{1}$ University of Poitiers \\ ${ }^{2}$ Université de Poitiers \\ 3 university of Poitiers \\ ${ }^{4}$ Universitie Paris-Saclay \\ ${ }^{5}$ CEA, Universitie Paris-Saclay \\ ${ }^{6}$ UMR 6269 CNRS INSU - Université de Poitiers \\ Presenting Author: ali.asaad@univ-poitiers.fr
}

The effect of particles organization on the diffusion of tracers in clayey porous media was investigated by Van Loon et al. 2004 [1] who reported that the effective diffusion coefficient $\left(D_{e}\right)$ of water tracer measured parallelly to the bedding was 6 times higher than value measured perpendicularly. Recently, Dabat et al. [2] succeeded in linking the anisotropy of water diffusion tensor to the clay particles preferential orientation in a noncharged clayey porous media with only interparticle porosity (kaolinite). However, such a quantitative link remains to be investigated for charged dual porosity clays. Hence, the present study investigates the role played by interparticle and interlayer porosities, and clay particles preferential orientation, on the diffusion of water in vermiculite porous media.

Na-vermiculite (high charge swelling clay) was chosen due to the absence of osmotic swelling of this mineral in water saturated conditions, allowing then to maintain the distribution between interparticle and interlayer porosities [3]. Through-diffusion (TD) experiments of water (HDO) were performed on two Navermiculite porous media, having the same total porosity of 0.49 (including 0.25 interparticle porosity), but different degrees of preferential orientation of the particles. The latter was quantified through the order parameter $<\mathrm{P}_{2}>$ obtained from X-ray scattering analyses [2], ranging from 0 to 1 for pure isotropic and anisotropic medium, respectively.

TD results show that $D_{e}$ of HDO is lowered by almost a factor 2 when $\left\langle\mathrm{P}_{2}>\right.$ increases from 0.03 to 0.41 for samples having the same total porosity. Furthermore, $D_{e}$ values obtained for vermiculite samples were very close to those obtained with kaolinite media having a total porosity of 0.25 (same interparticle porosity of vermiculite samples). This suggests that the flux of HDO diffusing through the interlayer porosity in the overall HDO flux traversing the samples is very marginal. Experimental results are also in fair agreement with Brownian Dynamic simulations carried out with analogous virtual porous media, which will be detailed in the presentation.

[1] Van Loon L. et al. (2004). EST, 38, 5721-5728.

[2] Dabat T. et al. (2020). Appl. Clay Sci., 184, 105354.

[3] Tertre E. et al. (2018). EST, 52, 1899-1907. 